TITLE:

\title{
Dynamics of cavitation bubbles generated by multi-pulse laser irradiation of a solid target in water
}

\section{$\operatorname{AUTHOR}(\mathrm{S}):$}

Tamura, Ayaka; Sakka, Tetsuo; Fukami, Kazuhiro; Ogata, Yukio H.

\section{CITATION:}

Tamura, Ayaka ... [et al]. Dynamics of cavitation bubbles generated by multi-pulse laser irradiation of a solid target in water. Applied Physics A 2013, 112(1): 209-213

\section{ISSUE DATE:}

2013-07

URL:

http://hdl.handle.net/2433/189886

\section{RIGHT:}

The final publication is available at Springer via http://dx.doi.org/10.1007/s00339-0127291-x; This is not the published version. Please cite only the published version.; この論文 は出版社版でありません。引用の際には出版社版をご確認ご利用ください。 


\title{
Dynamics of cavitation bubbles generated by multi-pulse laser irradiation of a solid target in water
}

\author{
Ayaka Tamura $\cdot$ Tetsuo Sakka $\cdot$ Kazuhiro Fukami $\cdot$ Yukio H. Ogata \\ Institute of Advanced Energy, Kyoto University, Gokasho, Uji-shi, Kyoto 611-0011, \\ Japan \\ e-mail: a-tamura@iae.kyoto-u.ac.jp \\ Fax: +81-774-38-3498
}

\begin{abstract}
The effect of the second and later pulses on the expansion dynamics of the cavitation bubble produced by multi-pulse microchip laser irradiation of a $\mathrm{Cu}$ target in water has been investigated. We clarified the bubble dynamics by taking shadowgraph images and measuring the bubble radius as a function of time. Shock waves were also measured to investigate the explosive expansion of the bubble. As a result, the second and later pulses did not cause an explosive expansion, and the ablation of the target by these pulses was rather mild, although they had a certain contribution to the expansion of the bubble. The energies given to the bubble expansion from the first pulse and also from the second pulse were estimated by comparing the experimental results with the calculation based on the Rayleigh model. The effect of the second and later pulses upon the bubble dynamics is discussed from the view point of the application to underwater laser-induced breakdown spectroscopy.
\end{abstract}




\section{Introduction}

Laser-induced breakdown spectroscopy (LIBS) can be a unique technique for in situ surface elemental analysis of solid targets submerged in water (underwater LIBS) and is expected to be applied in various purposes $[1,2]$. However, the emission spectra of the plasma produced by the underwater LIBS configuration suffer from intense continuum and extraordinary broadening due to the strong confinement effect of the plasma in water [1-4]. To improve the quality of the spectra in water, double pulse irradiation $[1,2$, 5] and long pulse irradiation [6] schemes have been proposed. Although these methods enable us to obtain clear atomic lines for underwater LIBS applications, the time-gated detection has been believed to be always necessary to avoid the continuum and extraordinary broadening, which arises immediately after the laser pulse irradiation $[1,2$, 7].

Recently, we have found that well-defined narrow atomic spectral lines without a continuum or an intense broadening can be obtained without relying on the time-gated detection by optimizing the laser power as well as the pulse interval in the double pulse and multi-pulse irradiation [8]. The energy of each pulse is as low as $0.5 \sim 1.0 \mathrm{~mJ}$. The first pulse produces a cavitation bubble without any appreciable emission in visible region, while the second pulse generates a comparatively low-density plasma which gives clear spectral lines. The bubble formation by the first pulse and the plasma formation in the bubble by the second pulse are essential to obtain well-defined atomic spectra without time-gating of the detection.

On the other hand, the effect of the second or later pulses on the bubble expansion dynamics in case of double pulse or multi-pulse irradiation is not clear so far. For underwater LIBS applications, it is desirable to make the plasma density quite low so that the deformation of the spectra is minimum [7]. Therefore, whether the bubble can expand further or not by controlling the pulse sequence is an important issue.

The aim of the present work is to clarify the effect of the second and later pulses on the bubble dynamics. Time-resolved shadowgraph images have been taken using an imaging system with nanosecond time-resolution, and the cavitation bubble radius was obtained as a function of time. The results were compared with theoretical calculation, and the effects of the second and later pulses upon the bubble dynamics are 
discussed.

\section{Experimental}

A Q-switched Nd:YAG multi-pulse microchip laser with a wavelength of $1064 \mathrm{~nm}$ provided from Hamamatsu Photonics Inc. was used as an excitation source. The laser provides 10-12 pulses in one sequence with the pulse interval of $\sim 18 \mu$ s. Each pulse has the width of $3 \mathrm{~ns}$ and the pulse energy of $\sim 0.5 \mathrm{~mJ}$. The total energy of one sequence of the laser shot was $\sim 6.0 \mathrm{~mJ}$. Figure 1 shows an example of the time profile of the multi-pulse microchip laser obtained with a silicon-PIN photodiode. The timing of each pulse varies shot to shot and also shows a long term drift. Earlier pulses vary by several microseconds, while the variation is accumulated to several tens of microseconds for the later pulses.

Experimental setup for the shadowgraph measurement is shown in Fig. 2. The laser beam was focused onto a target from the normal direction by a $60-\mathrm{mm}$ focal length lens. A Cu plate used as a target was placed in a cell filled with pure water. The cell is made of polytrifluoroethylene resin, and has a hole to insert a water-immersion objective lens from the side wall. The magnification of this lens was 20x, and the spatial resolution was as high as $0.64 \mu \mathrm{m}$ per pixel. The laser irradiation spot was illuminated by a tungsten lamp from behind, and the images of the bubble were obtained by shadowgraphy. Time-resolved shadowgraph images were taken by an intensified charge coupled device (ICCD) camera and obtained at various gate delay times. The gate width of the ICCD was $500 \mathrm{~ns}$.

Shock waves generated by the multi-pulse irradiation were measured by using a Müller-Platte Needle Probe (Dr. Müller Instruments Co.). The probe was placed in water at a distance of $\sim 2 \mathrm{~mm}$ from the irradiation spot. The signal of the shock wave was recorded by an oscilloscope. To check the timing of the shock wave generation, the time profile of the multi-pulse microchip laser was monitored simultaneously with a silicon-PIN photodiode.

\section{Results}

Figure 3 shows shadowgraph images obtained at various ICCD delay times after the 
first pulse irradiation. Time sequence of the pulses and shadowgraphs, (a) - (j) is as follows, 1st pulse, (a), (b), 2nd pulse, (c), 3rd pulse, 4th pulse, (d), 5th pulse, (e), 6th pulse, (f), (g), 7th pulse, 8th pulse, (h), 9th pulse, 10th pulse, (i) and (j). The cavitation bubble produced by the first pulse expanded with time. After the second pulse irradiation (Fig. 3(c)), the bubble still grew. A small bubble was sometimes generated by the third or later pulse irradiation on the top of the bubble already produced (Fig. 3(e), (f)). The bubble deformed seriously after the sixth pulse irradiation (Fig. 3(g), (h)).

Figure 4 shows the temporal variation of the bubble radius measured in the direction perpendicular to the target surface. When the bubble deformed seriously, the bubble radius is not well-defined. The bubble size increased after the first and second pulse irradiation. But it remained a certain size after the third pulse until the fifth pulse irradiation, and then it shrunk. The rate of the bubble expansion seems to increase slightly but discontinuously at the timing of the second pulse and probably also at the third and forth pulses. The solution of the Rayleigh-Plesset equation best-fitted to experimental results is also shown in Fig. 4, which will be discussed in detail later.

The result of shock waves measurement as well as the laser profile is shown in Fig. 5. The first shock wave signal was observed $1.4 \mu$ s after the first pulse, meaning that the generation of the shock wave was at the timing of the first pulse irradiation. The second shock wave signal corresponds to the bubble collapse. We did not observe any shock wave signal after the second one. Also, the shock wave was not generated at the timing of the second and later pulses.

\section{Discussion}

It is clearly observed in Figs. 3 and 4 that the bubble shrinks and collapses even during the irradiation of the sequential pulses. From around $70 \mu \mathrm{s}$, the boundary between the bubble and the liquid became unstable, and the bubble could not remain hemispherical or deformed in a random manner. Oscillation in the bubble size, i.e., the repeating of the formation and collapse of the bubble, which were often observed in previous studies [9-12] were not observed in the present work. This behavior agrees with the results that no shock wave signal was observed after the second one. It seems that these results are due to a very low pulse energy employed in the present work. Also, the results show that 
the shock wave was not observed at the timing of the second or later pulses. This means that the second and later pulses do not produce an abrupt change of the pressure in the bubble. From the viewpoint of the shock wave generation, the behavior of the bubble produced by multi-pulse laser irradiation seems to be quite similar to the one produced by a single pulse [13].

It has been known that the Rayleigh model [14] describes a collapse of the cavitation bubble in an infinite and incompressible fluid with the assumption that the pressure of the liquid as well as the pressure inside the bubble is constant during the bubble collapse $[10,11,13]$. In the Rayleigh model the radius of the bubble at delay time $t$ after the pulse irradiation, $R(t)$, is described by $[10,11]$

$$
R(t) \frac{d^{2} R(t)}{d t^{2}}+\frac{3}{2}\left[\frac{d R(t)}{d t}\right]^{2}=\frac{P_{\infty}}{\rho_{\mathrm{L}}},
$$

where $P_{\infty}$ is the liquid pressure (ambient pressure), and $\rho_{\mathrm{L}}$ is the liquid density. The velocity of the bubble boundary $U$ obtained by integrating Eq. (1) is given by $[10,11$, $14]$

$$
U^{2}=\left(\frac{d R}{d t}\right)^{2}=\frac{2 P_{\infty}}{3 \rho_{\mathrm{L}}}\left(\frac{R_{\max }^{3}}{R^{3}}-1\right) .
$$

Here, $R_{\max }$ is the maximum radius of the bubble. The integral form of Eq. (2) is as follows

$$
t=\sqrt{\frac{3 \rho_{\mathrm{L}}}{2 P_{\infty}}} \int_{0}^{R} \frac{\left(r / R_{\max }\right)^{3 / 2}}{\left(1-\left(r / R_{\max }\right)^{3}\right)^{1 / 2}} d r .
$$

By using this formula $R$ as a function of $t$ is calculated numerically using the trapezoidal integration method. We fitted this calculation to experimental results, and obtained the best-fit maximum radius, $R_{\max }$, of the bubble. In the calculation $P_{\infty}=10^{5} \mathrm{~Pa}$ and $\rho_{\mathrm{L}}=10^{3}$ $\mathrm{kg} \mathrm{m}{ }^{-3}$ were used. Figure 6 shows the comparison between the experimental observation and the best-fit result of the Rayleigh model. $R_{\max 1}$ is the maximum radius obtained by fitting to the data after the first pulse before the second pulse, and $R_{\max 2}$ is that obtained using the data after the second pulse before the third pulse. The Rayleigh model was in accordance with the experimental results of the bubble radius. The maximum radii of the bubbles obtained as the best-fit parameter values of the Rayleigh 
model were 586.4 and $657.1 \mu \mathrm{m}$ for $R_{\max 1}$ and $R_{\max 2}$, respectively. This means that the bubble behavior of the multi-pulse irradiation is slightly different from that of the single pulse irradiation.

The energy $E_{\mathrm{B}}$ of a cavitation bubble is proportional to the cube of its maximum radius $[10,11,15,16]$

$$
E_{B}=\frac{4 \pi P_{\infty}}{3} R^{3}{ }_{\max } .
$$

By substituting the maximum radius obtained above into Eq. (4), the energies of the bubbles converted from the first and second pulse energies were calculated to be 84.5 and $34.4 \mu \mathrm{J}$, respectively. The bubble energy transferred from the second pulse is only $41 \%$ of that from the first pulse. This suggests that the contribution of the second pulse energy converted into something other than the bubble expansion or the kinetic energy of the bubble expansion is larger than that of the first pulse. Although it is not clear what the pulse energies are used for, possible candidates may be the plasma formation, heating of the surface and/or inside the bubble, evaporation of the surface species, and so on [17]. The present result of the shock wave measurements suggests that the second and later pulse irradiation cannot raise the pressure inside the bubble to give an explosive expansion. In other words, the energies of the second and later pulses are only slightly converted into the bubble expansion energy. These results obtained by considering the Rayleigh model agree with the results of the shock wave measurement.

For further examination for the verification of this idea, we introduce the Rayleigh-Plesset equation to describe the bubble dynamics. It is well known that the temporal variation of the spherical bubble radius produced by a single pulse irradiation is in good agreement with the Rayleigh-Plesset equation [12, 18, 19]. Unlike the Rayleigh model, the Rayleigh-Plesset equation takes account of the temporal variation of the pressure inside the bubble. To investigate the effect of the second and later pulses, the dynamics of the bubble was studied theoretically by fitting to the Rayleigh-Plesset equation. The Rayleigh-Plesset equation is given in the following form $[12,18,19]$

$$
\frac{P_{\mathrm{B}}(t)-P_{\infty}}{\rho_{\mathrm{L}}}=R(t) \frac{d^{2} R(t)}{d t^{2}}+\frac{3}{2}\left[\frac{d R(t)}{d t}\right]^{2}+\frac{4 v}{R(t)} \frac{d R(t)}{d t}+\frac{2 S}{\rho_{\mathrm{L}} R(t)},
$$

where $P_{\mathrm{B}}(t)$ is the pressure inside the bubble, and $\rho_{\mathrm{L}}, v$, and $S$ are the density, the kinematic viscosity coefficient, and the surface tension of water, respectively. Assuming 
an adiabatic van der Waals equation of state for a spherical bubble, the vapor pressure inside the bubble is given by [19]

$$
P_{\mathrm{B}}(t)=\left(P_{\infty}+\frac{2 S}{R}\right)\left(\frac{R_{\infty}^{3}-h^{3}}{R^{3}-h^{3}}\right)^{\gamma} .
$$

Here $R_{\infty}$ is the radius at which the pressure inside the bubble corresponds to $P_{\infty}, h$ is the radius determined by the excluded volume of the water molecules and $\gamma$ is the ratio of the specific heats. In the present work, $h=R_{\infty} / 9.174$ and $\gamma=1.13$ have been used [19].

The solid line in Fig. 4 shows the solution of the Rayleigh-Plesset equation best-fitted to the bubble radius after the first pulse irradiation before the second pulse. Good agreement was obtained for the fitting in this time range. The difference between the bubble radius expected from the Rayleigh-Plesset calculation best-fitted to the data before the second pulse and the experimental observation is rather small. On the other hand, the maximum size of the bubble after the second and later pulses became slightly larger than that expected for the bubble produced only by the first pulse. These results suggest that the second pulse irradiation has limited effects on the bubble expansion.

The present results give important insights into the mechanism of low-density plasma generation by the multi-pulse laser. Such a low-density plasma gives well-defined atomic spectra without a continuum or a significant broadening, even by the non-gated detection. Our recent study using a multi-pulse laser suggested that the role of the second pulse is to produce a plasma in the bubble rather than the expansion of the bubble, while the role of the first pulse is to produce the bubble [8]. This agrees with the present result that the second pulse irradiation makes a mild ablation which does not give an explosive expansion nor a shock wave. Such a mild ablation is important to produce the low-density plasma applicable to underwater LIBS measurement without time-gated detection.

\section{Conclusions}

We investigated the expansion dynamics of the cavitation bubble generated by the multi-pulse laser irradiation of a solid target immersed in water. It has been clarified that the second and later pulses did not cause an explosive expansion of the bubble having been generated by the first pulse, although additional slight expansion was observed. It 
was found that the second pulse contributes to the bubble expansion only about $28 \%$ of the first pulse. If the remaining part of the second pulse energy was used to produce the plasma in the bubble, the energies of the first and the second pulses are used in quite different ways, i. e., the first pulse produces a bubble and the second pulse produces a plasma in the bubble. Such a difference in the roles of the first pulse and the second pulse seems to be essential to keep the density of the light emitting region low and to avoid the generation of a high-density plasma. As a result, such behavior enables us to obtain well-defined atomic spectra without a continuum or a significant broadening even without the time-gated detection in the underwater LIBS. 


\section{References}

1. A. E. Pichahchy, D. A. Cremers, M. J. Ferris, Spectrochim. Acta B 52, 25 (1997)

2. A. De Giacomo, M. Dell'Aglio, O. De Pascale, M. Capitelli, Spectrochim. Acta B 62, $721(2007)$

3. T. Sakka, S. Iwanaga, Y. H. Ogata, A. Matsunawa, T. Takemoto, J. Chem. Phys. 112, 8645 (2000)

4. T. Sakka, K. Takatani, Y. H. Ogata, M. Mabuchi, J. Phys. D 35, 65 (2002)

5. R. Nyga, W. Neu, Opt. Lett. 18, 747 (1993)

6. T. Sakka, H. Oguchi, S. Masai, K. Hirata, Y. H. Ogata, M. Saeki, H. Ohba, Appl. Phys. Lett. 88, 061120 (2006)

7. H. Oguchi, T. Sakka, Y. H. Ogata, J. Appl. Phys. 102, 023306 (2007)

8. T. Sakka, A. Tamura, T. Nakajima, K. Fukami, Y. H. Ogata, to be published

9. X. Chen, R.Q. Xu, J.P. Chen, Z.H. Shen, J. Lu, X.W. Ni, Appl. Opt. 43, 3251 (2004)

10. R. Petkovšek, P. Gregorčič, J. Appl. Phys. 102, 044909 (2007)

11. B. Li, H. Zhang, J. Lu, X. Ni, Opt. Laser Technol. 43, 1499 (2011)

12. W. Soliman, T. Nakano, N. Takada, K. Sasaki, Jpn. J. Appl. Phys. 49, 116202 (2010)

13. T. Tsuji, Y. Okazaki, Y. Tsuboi, M. Tsuji, Jpn. J. Appl. Phys. 46, 1533 (2007).

14. L. Rayleigh, Philos. Mag. 34, 94 (1917).

15. A. Vogel, J. Noack, K. Nahen, D. Theisen, S. Busch, U. Parlitz, D. X. Hammer, G. D. Noojin, B. A. Rockwell, R. Birngruber, Appl. Phys. B 68, 271 (1999)

16. S. V. Egerev, Acoust. Phys. 49, 59 (2003)

17. M. von Allman, A. Blatter, Laser-Beam Interactions with Materials, Physical Principles and Applications, 2nd ed., (Springer Verlag, Berlin, 1994)

18. C. Brennen, Cavitation and Bubble Dynamics, Chap. 2 (Oxford University Press, Oxford, U.K., 1995)

19. A. Casavola, A. De Giacomo, M. Dell'Aglio, F. Taccogna, G. Colonna, O. De Pascale, S. Longo, Spectrochim. Acta B 60, 975 (2005) 
Figure captions

Fig. 1

Time profile of the multi-pulse microchip laser. The profile of the first pulse magnified 25000 times in the time axis is shown in the inset.

Fig. 2

Experimental set up for the shadowgraph measurement.

Fig. 3

Shadowgraphs of the bubble produced by the multi-pulse laser ablation of a $\mathrm{Cu}$ target in water at various ICCD delay times from the first pulse irradiation. The timing of the measurement is given in each shadowgraph as a delay from the first pulse.

Fig. 4

The radius of the bubble produced by the irradiation of a $\mathrm{Cu}$ target in water plotted as a function of the ICCD delay time from the first pulse irradiation (open circle). The solution of the Rayleigh-Plesset equation best-fitted to the experimental data before the second pulse is also shown (solid line). The figures at the top show the pulse numbers.

Fig. 5

Shock wave signals (a) and the time profile of the laser sequence (b).

Fig. 6

Comparison between the experimental observation of the bubble radius (cross symbol) and the solution of the Rayleigh model (solid line) best-fitted to the data after the first pulse (a) and after the second pulse (b). 


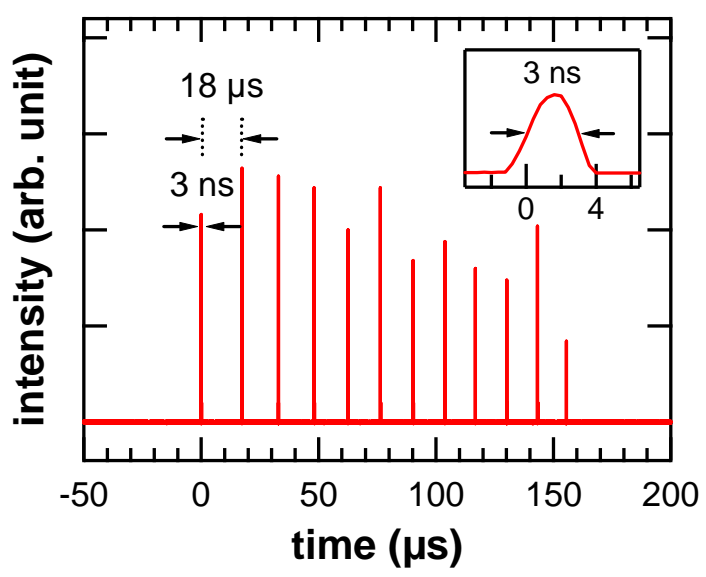

Fig. 1 


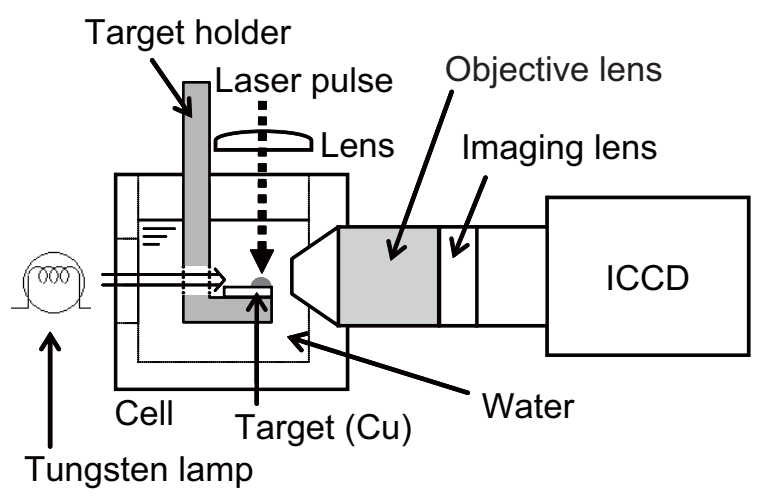

Fig. 2 


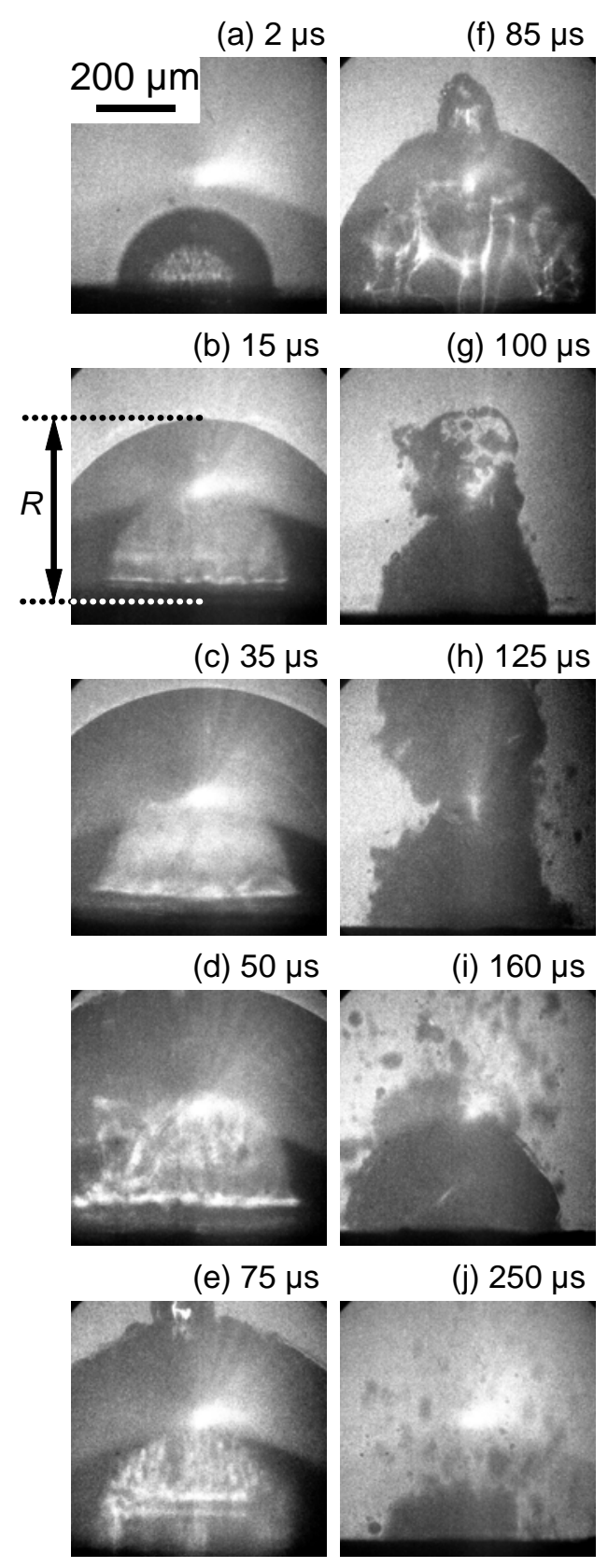

Fig. 3 


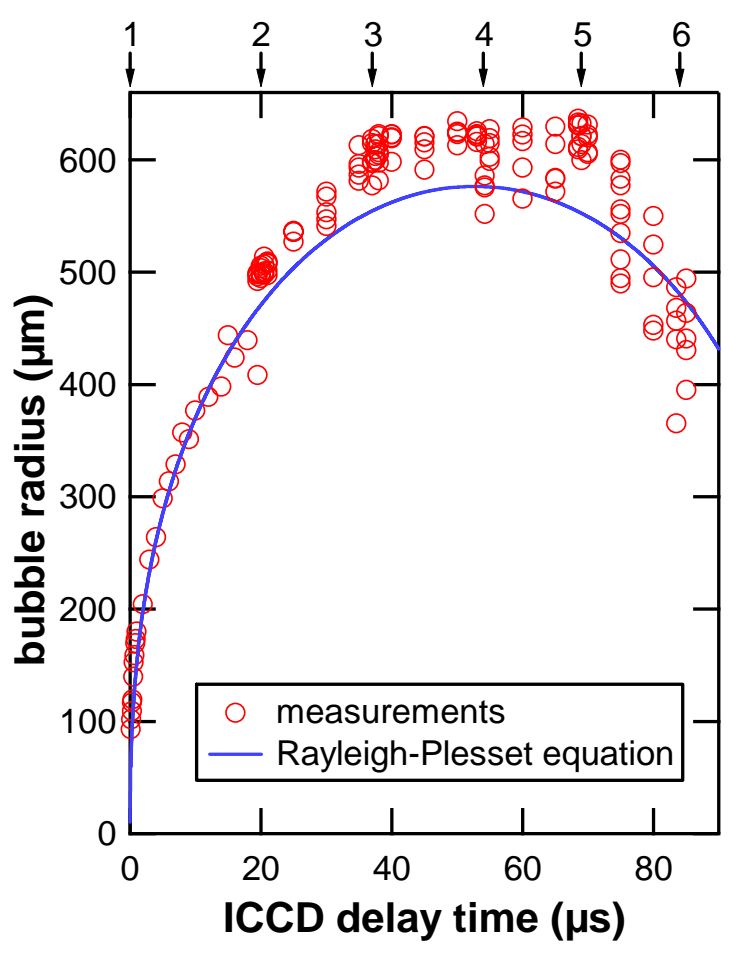

Fig. 4 


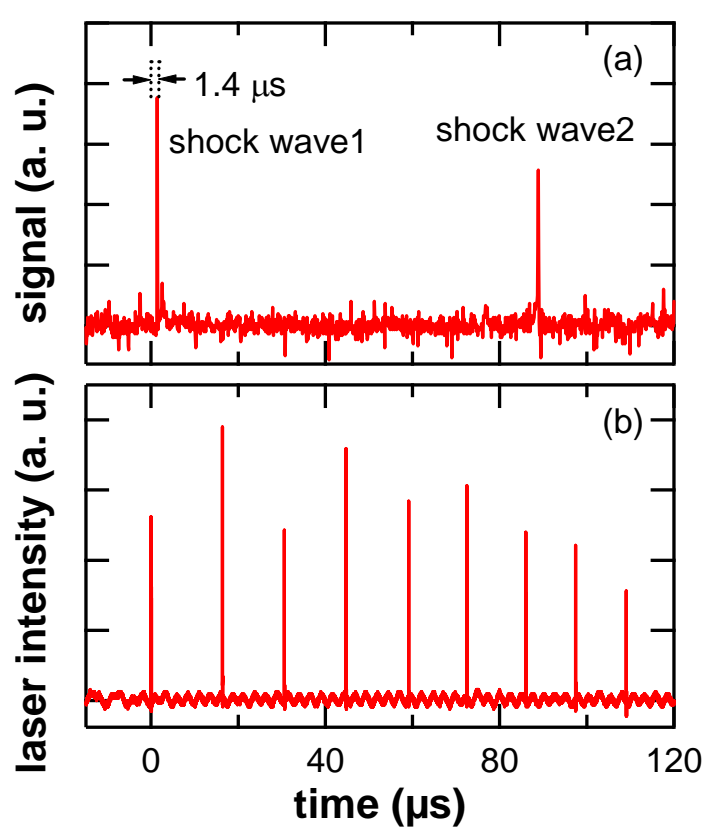

Fig. 5 

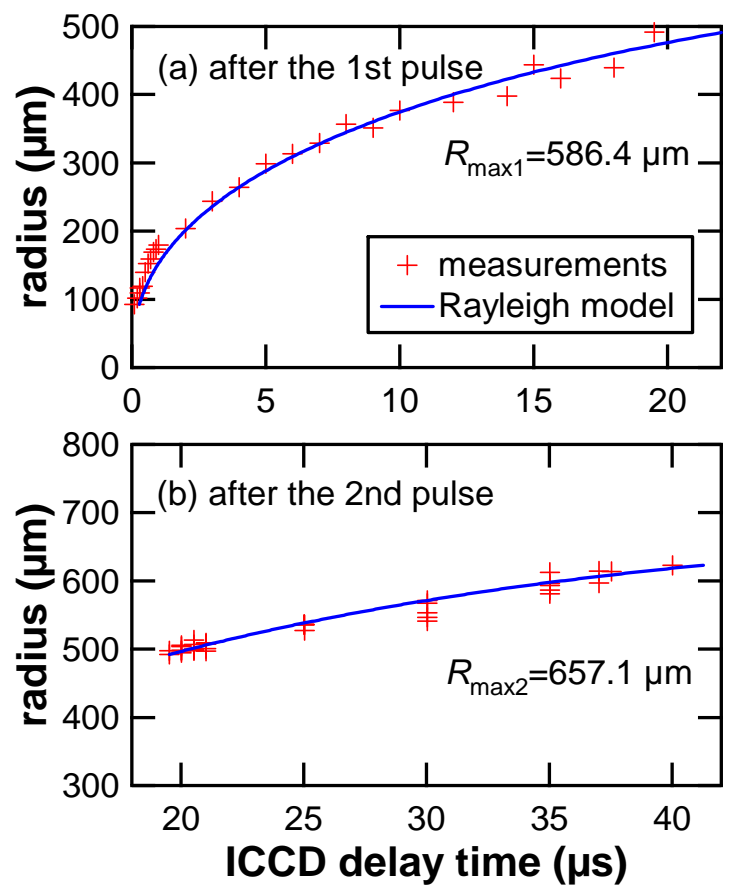

Fig. 6 